\title{
Development of a coronal mass ejection arrival time forecasting system using interplanetary scintillation observations
}

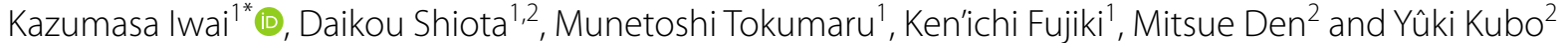

\begin{abstract}
Coronal mass ejections (CMEs) cause disturbances in the environment of the Earth when they arrive at the Earth. However, the prediction of the arrival of CMEs still remains a challenge. We have developed an interplanetary scintillation (IPS) estimation system based on a global magnetohydrodynamic (MHD) simulation of the inner heliosphere to predict the arrival time of CMEs. In this system, the initial speed of a CME is roughly derived from white-light coronagraph observations. Then, the propagation of the CME is calculated by a global MHD simulation. The IPS response is estimated by the three-dimensional density distribution of the inner heliosphere derived from the MHD simulation. The simulated IPS response is compared with the actual IPS observations made by the Institute for Space-Earth Environmental Research, Nagoya University, and shows good agreement with that observed. We demonstrated how the simulation system works using a halo CME event generated by a X9.3 flare observed on September 5, 2017. We find that the CME simulation that best estimates the IPS observation can more accurately predict the time of arrival of the CME at the Earth. These results suggest that the accuracy of the CME arrival time can be improved if our current MHD simulations include IPS data.
\end{abstract}

Keywords: Interplanetary scintillation, Coronal mass ejection, Space weather forecasting, Magnetohydrodynamics, Data assimilation

\section{Introduction}

Solar wind and coronal mass ejections (CMEs) sometimes cause geospace disturbances when they arrive at the Earth. These disturbances are closely related to social infrastructures such as radio telecommunications, spacecraft and aircraft operation, and global positioning system (GPS)-based navigation. CME occurrences around the Sun have been observed using space-based white-light coronagraphs (e.g., Yashiro et al. 2004). However, the initial speeds of CMEs derived from white-light coronagraph observations are ambiguous because of line-of-sight projection effects. In addition, the acceleration and deceleration processes of CMEs propagating through interplanetary space are not well understood.

\footnotetext{
*Correspondence: k.iwai@isee.nagoya-u.ac.jp

${ }^{1}$ Institute for Space-Earth Environmental Research, Nagoya University,

Furo-cho, Chikusa-ku, Nagoya 464-8601, Japan

Full list of author information is available at the end of the article
}

These factors make the prediction of CME arrival difficult. There have been many models used to estimate the propagation of the CMEs. These include kinematic models and MHD simulations (Chen 1996; Gopalswamy et al. 2001; Vršnak and Gopalswamy 2002; Odstrcil 2003; Cargill 2004; Shiota and Kataoka 2016). There are also some studies that predict the CME arrival time using space-based remote sensing and in situ observations of the inner heliosphere such as Solar TErrestrial RElations Observatory (STEREO) and MESSENGER spacecraft (e.g., Rollett et al. 2016; Möstl et al. 2017). Although the observations from these spacecrafts give important information about the propagation of CMEs in the inner heliosphere, their data are only available over limited time periods.

The solar wind includes a density disturbance, which causes the scattering of radio waves; this phenomenon is called interplanetary scintillation (IPS). Interplanetary CMEs (ICMEs) cause an increase in the amplitude of 
IPS because the sheath region, i.e., the high density and turbulent region in front of the CME, can significantly scatter radio emissions. Propagating CMEs have been observed by IPS observations (Tokumaru et al. 2000, 2003; Manoharan 2006; Iju et al. 2013, 2014; Glyantsev et al. 2015). In these previous studies, the CME propagation speed was derived from CME locations, which are determined from the radio source locations in the sky, and the time period that elapses between the CME occurrence near the solar surface and its IPS observation. Typical IPS telescopes scan the inner heliosphere once a day, making it difficult to detect the front of fast-propagating CMEs more than one time. Only a few campaignbased observations have scanned the sky twice a day (e.g., Johri and Manoharan 2016) in order to determine CME locations at higher than a daily cadence.

The IPS amplitude has been estimated using solar wind models, and the solar wind itself has been re-constructed via the IPS tomography technique (Jackson et al. 1998; Kojima et al. 1998). CMEs are not prominent in the IPS tomography presented in these previous articles, which reconstruct the heliosphere assuming it corotates. However, a more recent time-dependent tomography analysis (Jackson et al. 2003; Jackson et al. 2011 and references therein) can provide CME information and is also used as an inner boundary for the three-dimensional (3D) MHD simulations for forecasting CME structure ( $\mathrm{Yu}$ et al. 2015) and arrival (Jackson et al. 2015). Tokumaru et al. $(2003,2006)$ have also used a CME model to fit the IPS observations. This model-fitting technique requires only one day of IPS data, and it provides the 3D structure of CMEs in interplanetary space. The propagation of CMEs in interplanetary space is affected by the background solar wind. Therefore, a CME-solar wind model that integrates both the effects of background solar wind and CMEs (e.g., Chen 1996; Vršnak and Gopalswamy 2002) is important to accurately forecast their arrival at the Earth using IPS observations.

The global MHD simulations of the solar wind, which include the CMEs (e.g., Odstrcil 2003; Shiota and Kataoka 2016), enable us to estimate CME propagation and their interactions with background solar wind. These simulations provide the time variation of the electron density distributions of the inner heliosphere; knowledge of this variation enables us to estimate the scintillations of the radio emission.

The purpose of this study is to develop a system based on IPS analysis and global MHD simulations that can understand and refine the propagation processes of CMEs in the inner heliosphere and predict their arrival to the Earth more accurately than at the present. In our MHD simulation system, the propagation of the CME is calculated by the Space-weather-forecast-Usable System
Anchored by Numerical Operations and Observations (SUSANOO)-CME (Shiota and Kataoka 2016), and the IPS amplitude of each radio source is calculated using the 3D electron density variation derived from the MHD simulation. The estimated IPS response is compared with the observed IPS values to evaluate the accuracy of the MHD simulations.

\section{Methods \\ IPS observation}

The IPS observations are carried out by Institute for Space-Earth Environmental Research (ISEE), Nagoya University, Japan. ISEE operates radio telescopes dedicated to IPS observations at a radio frequency of $327 \mathrm{MHz}$. At this frequency, the IPS response under typical solar wind conditions can be approximated by weak scattering theory (Young 1971) between 0.2 and 1.0 astronomical units (AU). IPS data obtained by the Solar Wind Imaging Facility (SWIFT: Tokumaru et al. 2011) radio telescope of ISEE at the Toyokawa Observatory were employed in this study. This system has a fixed cylindrical parabolic reflector of dimensions $108 \times 38 \mathrm{~m}$, which has a single beam steerable between $60^{\circ} \mathrm{S}$ and $30^{\circ} \mathrm{N}$ along the local meridian, and observes 50 to 70 radio sources each day throughout the year.

The data processing of our IPS observations is described in reports of Tokumaru et al. (2000, 2003). We usually derive a scintillation level for each radio source which is the amplitude ratio of the scintillating component and noise component of the power spectra. Then, we derive the ratio of the instantaneous scintillation level to the typical scintillation level which corresponds to the $g$ value (Gapper et al. 1982) allowing the detection of the IPS enhancement caused by transient phenomena such as CMEs.

\section{MHD simulation (SUSANOO-CME)}

The MHD simulation in this study was originally developed by Shiota et al. (2014) and further expanded by Shiota and Kataoka (2016). This numerical code simulates the inner heliosphere between 25 and 425 solar radii (Rs) using a Yin-Yang grid (Kageyama and Sato 2004). The magnetic field at the inner boundary is derived from the potential field source surface (PSFF) model. The velocity, density and temperature are derived from empirical models of the solar wind (Arge and Pizzo 2000; Hayashi et al. 2003). CMEs are included in the inner boundary of the simulation as spheromak-type magnetic flux ropes (SUSANOOCME: Shiota and Kataoka 2016). The initial velocities of CMEs are roughly derived semiautomatically from the data of the Large Angle and Spectrometric Coronagraph (LASCO: Brueckner et al. 1995) onboard the 
Solar and Heliospheric Observatory (SOHO). For this study, several CME velocities within the error range are simulated to form an ensemble simulation set (Shiota et al. in prep). These simulations are evaluated using the IPS data, as described in the next section.

\section{IPS estimation using the MHD simulation}

We estimated the IPS $g$ value using the theoretical expressions of the radio scintillation assuming a weak scattering condition as given by Young (1971). The scintillation index (or so-called $m$-index) is the amplitude ratio of the total radio emission to the scintillating component of the radio emission. This index is obtained by integrating the density fluctuation of the solar wind $\left(\Delta N_{e}\right)$ convolved with a weighting function of the IPS $w(z)$ along the line of sight to the radio source:

$$
m^{2} \propto \int_{0}^{\infty} \Delta N_{e}^{2} w(z) \mathrm{d} z
$$

where $z$ is the distance along the line of sight. The weighting function of the IPS is given by

$$
w(z)=\int_{0}^{\infty} k^{1-q} \sin ^{2}\left(\frac{k^{2} z \lambda}{4 \pi}\right) \exp \left(-\frac{k^{2} z^{2} \psi^{2}}{2}\right) \mathrm{d} k,
$$

where $k, q, \lambda$ and $\psi$ are the wave number of density fluctuations in the solar wind, spectral index of the density disturbance, wavelength of the radio emission $(0.92 \mathrm{~m})$ and apparent size of the observed radio source. We set $q=11 / 3$ because the density disturbance of the solar wind is shown to follow the Kolmogorov spectrum in the inner heliosphere (Woo and Armstrong 1979). Following Tokumaru et al. (2003), we assumed all radio sources to be 0.1 arcsec in size. The disturbance level of the solar wind is assumed proportional to density $\left(\Delta N_{e} \propto N_{e}\right)$. In addition, we assume the plasma density and election density have the same proportionality in our MHD simulation (i.e., electrical neutrality). Thus, we can estimate the scintillation index of radio sources from the total electron density along the line of sight by the following steps:

- Perform the MHD simulation as explained in the MHD simulation section but without the spheromak to obtain a time series of the 3D density distribution of the background solar wind.

- Estimate the scintillation index for a given radio source line of sight by integrating the convolution of the simulated electron density and the weighting function given by Eq. 2, which we expect to correspond to the index from the background solar wind.
- Perform the MHD simulation with CMEs to obtain a time series of the density distribution of the inner heliosphere using SUSANOO-CME.

- Estimate the scintillation index using the density distribution with the weighting function along the line of sight from the combined background solar wind and CMEs.

- Normalize the simulated scintillation index from the solar wind with CMEs to that simulated from the solar wind without CMEs. This normalized scintillation index gives the IPS $g$ values in our MHD simulation.

We compare the simulated IPS $g$ values with the observed IPS $g$ values and discuss this comparison in the following sections.

In the proposed system, we perform several simulations with different CME velocities and estimated IPS distributions from each simulation. Finally, the CME simulation that best reconstructs the IPS observation is used to forecast the CME arrival time.

\section{Results}

We demonstrate our IPS-MHD simulation using a halo CME event generated by a X9.3 flare observed on September 5, 2017 (e.g., Shen et al. 2018). The detailed analysis of the IPS data during this period is given by Tokumaru et al. (in prep). In this paper, we present the observed data and the derivation of the CME velocity from the viewpoint of space weather forecasting.

\section{IPS Observations}

The left panel of Fig. 1 shows a difference image from the $\mathrm{SOHO} / \mathrm{LASCO}$ white-light coronagraph on September 6 , 2017. The halo CME associated with the X9.3 flare is observed most prominently toward the south and west side of the Sun. The right panel in Fig. 1 shows an all-sky map of the IPS $g$ value observed by the SWIFT radio telescope. In this figure, the location of each radio source is shown at a distance from the Sun scaled to the closest point on the line of sight to the radio source (the so-called P-point). Hence, distance $D$ is expressed as $D=1 \mathrm{AU} \times \sin \theta$, where $\theta$ is the elongation of the radio source. The center of the figure is the Sun, and the largest circle indicates $1 \mathrm{AU}$ (the location of the Earth). The meridian scan observation of SWIFT started around 18 UT on September 6 from the west edge (right side) of the figure and ended around 10 UT on September 7 at the east edge (left side). Red, green and blue diamonds indicate the radio sources for which the $g$ value is larger than 2.0, between 1.5 and 2.0, and between 1.2 and 1.5, respectively. The red and green diamonds on the southeast region indicate the location of the CME. 

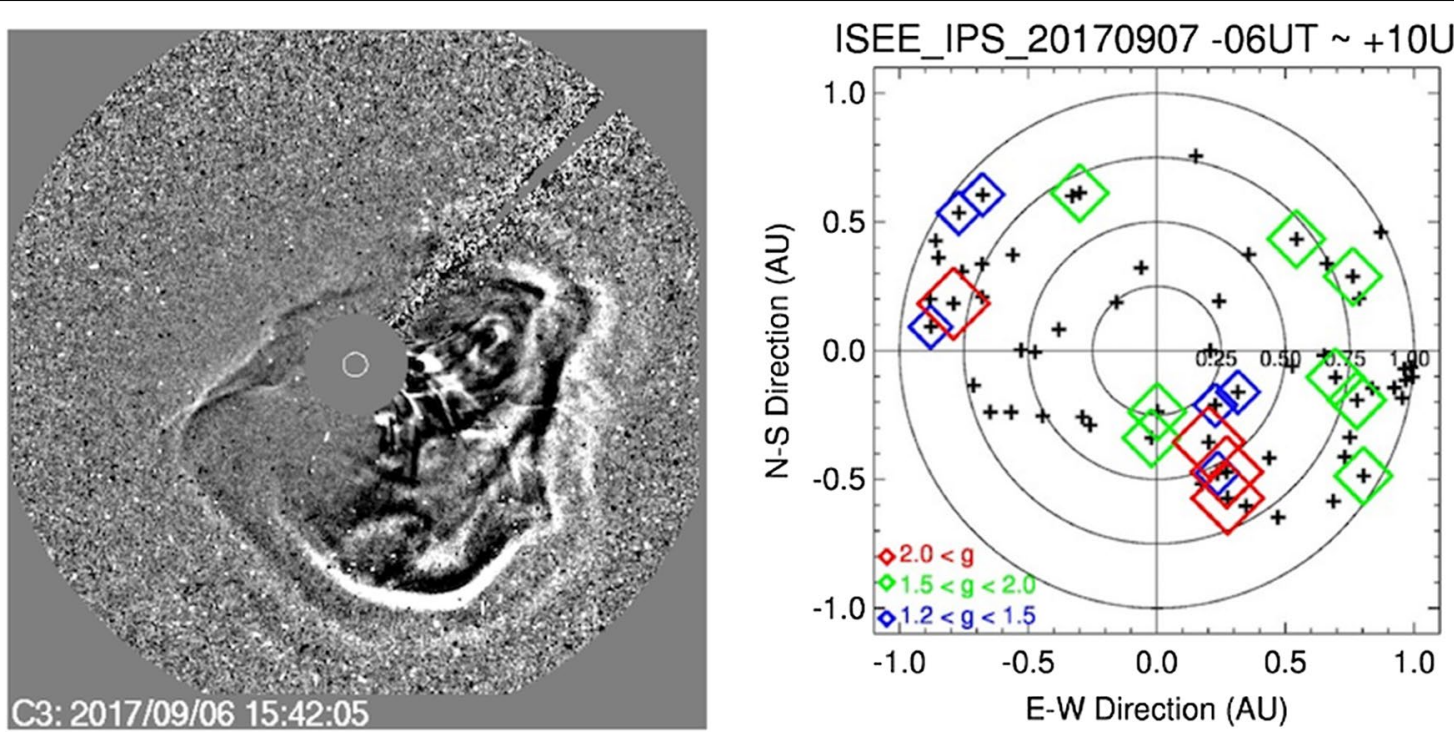

Fig. 1 (Left) An example of the white-light coronagraph image observed by SOHO/LASCO on September 6, 2017. (Right) An all-sky map of the IPS $g$ value observed by SWIFT on September 7, 2017. The symbols indicate +: all observed radio sources, diamonds: radio sources with $g$ values as follows: $2.0<g$ (red), $1.5<g<2.0$ (green) and $1.2<g<1.5$ (blue)

\section{MHD simulation and IPS estimation}

Figure 2a shows the scintillation index of the background solar wind estimated by SUSANOO. Figure $2 \mathrm{~b}$ shows the estimated scintillation index by SUSANOO that includes the CMEs that occurred in early September 2017. The ratio of the simulated indices shown in Fig. $2 \mathrm{~b}$ relative to those shown in Fig. 2a gives an estimation of the $g$ values, which are plotted in Fig. 2c. The distribution of the estimated $g$ values is compared with those of the observed $g$ values (Fig. 2d) using the same coordinate system (apparent elongation from the Sun in radians). We assumed the size of all radio sources is 0.1 arcsec to calculate the scintillation level, the usual value ascribed to most scintillating sources observed at radio frequencies of $327 \mathrm{MHz}$ (e.g., Tokumaru et al. 2003). Although the estimated scintillation index may be different from the actual one because of this, the estimated $g$ value in Fig. 2c does not exhibit the source size effect because the ratio of the scintillation indices calculated with and without CMEs cancels this effect. Note that we assume each spheromak is directly related to the position of the flare onset where the spheromak extends outward radially. These assumptions may limit the accuracy of the predicted $g$ value distributions, because of the fact that the flare location itself is often not the location of the ejected material, and nonradial flow in the corona is often known to be present. The difference between the spheromak and the actual CME shape also causes the different $g$ value distributions. Although the detailed analysis of the CME deflection is beyond the scope of this study, it should be mentioned that Shiota and Kataoka (2016) pointed out the deflection of the spheromak in the MHD simulation can affect the CME predictions.

\section{Parameter survey of the CME speed}

We estimate the initial conditions of the CMEs from $\mathrm{SOHO} / \mathrm{LASCO}$ observations and include the corresponding spheromaks in the simulation. Two CMEs occurred in the period of interest-one was generated by a M5.5 flare on September 4 (the first CME) and the other, by a X9.3 flare on September 6 (the second CME). Table 1 summarizes the parameters of the two CMEs. Only the velocity of the second CME is a free parameter in this study.

We performed several simulations for the second CME with different initial velocities ranging from $1000 \mathrm{~km} / \mathrm{s}$ to $2500 \mathrm{~km} / \mathrm{s}$. In this paper, we consider four of these simulations: (RUN1) $1000 \mathrm{~km} / \mathrm{s}$, (RUN2) $1500 \mathrm{~km} / \mathrm{s}$, (RUN3) $2000 \mathrm{~km} / \mathrm{s}$ and (RUN4) $2500 \mathrm{~km} / \mathrm{s}$. Figure 3 shows the sky map of the IPS estimated from the four simulations. We are able to estimate the IPS for any time and any direction of interest because the global MHD simulation of SUSANOO provides the time variation of the 3D electron density between 25 and 425 Rs. On the other hand, in an actual IPS observation by ISEE, the sky is scanned using the diurnal motion of the radio sources. Therefore, we only overplot the actual IPS observed within $1 \mathrm{~h}$ of each simulated time. 

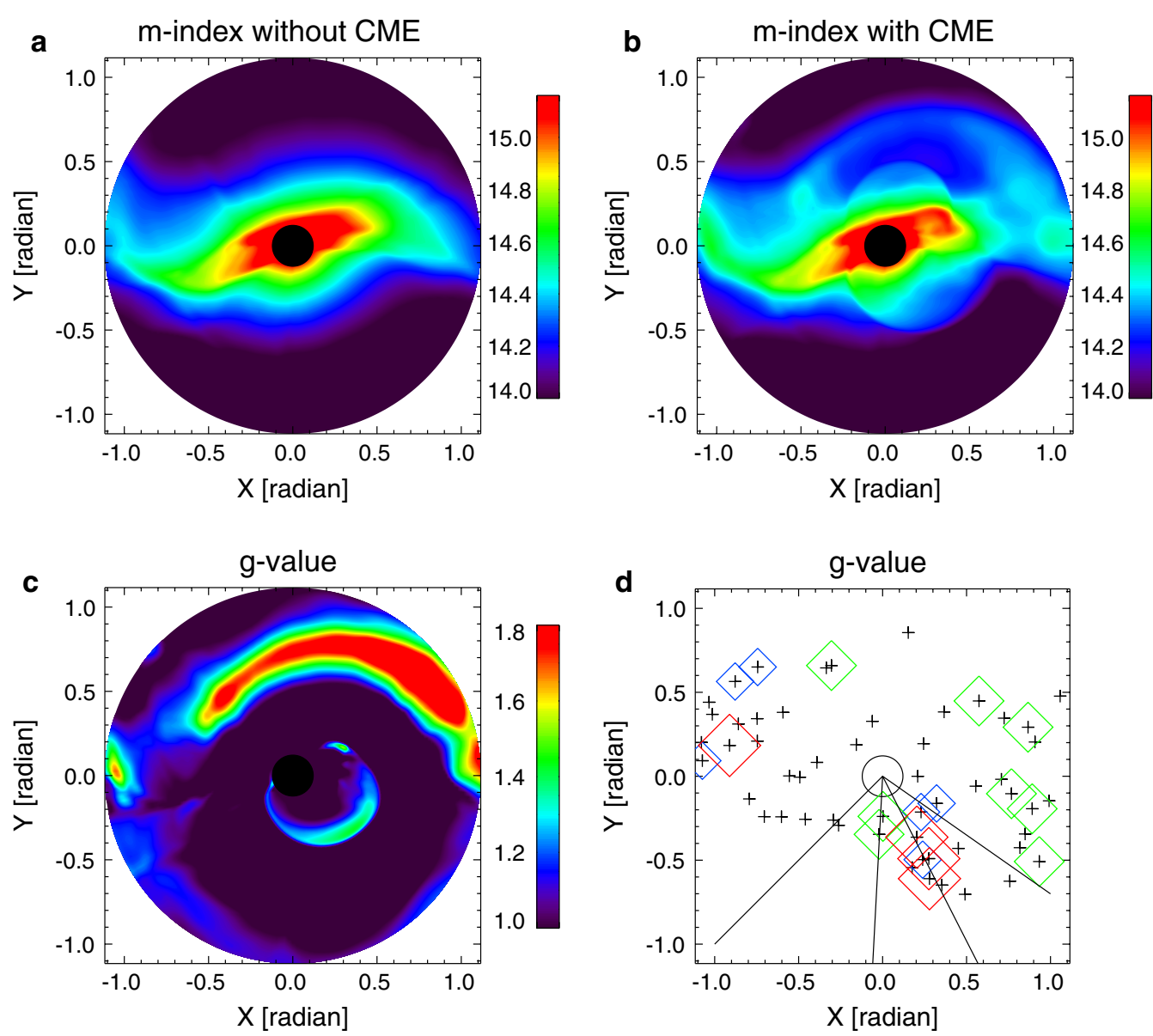

Fig. 2 Scintillation index and $g$ values estimated by SUSANOO and observed by SWIFT. a Scintillation index (log scale) of the background solar wind without CMEs estimated by SUSANOO at 1:00 UT on September 7, 2017. b Scintillation index (log scale) of the solar wind with CMEs estimated by SUSANOO-CME. c Estimated $g$ values, i.e., the ratio of the values shown in (b) to those shown in (a). d The IPS $g$ values observed by SWIFT displayed using the same coordinate system. Four solid lines indicate vertical axis of four panels in Fig. 5. The vertical and horizontal axes in the figure indicate the elongation from the Sun (in radians)

Table 1 Parameters of the spheromak included as the CME in the MHD simulation

\begin{tabular}{|c|c|c|c|c|c|c|c|}
\hline & Onset time & Velocity (km/s) & Longitude & Latitude & Width 1 & Width 2 & $B(M x)^{\mathrm{a}}$ \\
\hline First CME & 0904 20:28 & 1000 & $11^{\circ}$ & $5^{\circ}$ & $8^{\circ}$ & $60^{\circ}$ & $1.6 e+21$ \\
\hline Second CME & 0906 11:53 & $1000-2500$ & $23^{\circ}$ & $7^{\circ}$ & $3^{\circ}$ & $80^{\circ}$ & $3.0 e+21$ \\
\hline
\end{tabular}

a Total magnetic flux contained in the spheromak (Mx)

\section{Discussion}

\section{Physical meaning of the observed and estimated IPS} values

The observed $g$ value is derived by fitting the scintillation index over the source elongation. First, we derive a typical scintillation index curve at given elongation angle for each radio source by fitting the daily variation of the scintillation index using $\bar{m}=a D^{-b}$, where $a$ and $b$ are constants, and $D$ is the distance between the Sun and the closest point of the line of sight of the radio source. The ratio of $m$ and $\bar{m}$ at a given elongation angle is defined as the $g$ value. The enhancement of the observed $g$ value indicates a density enhancement along the line of sight by phenomena such as CMEs, heliospheric current sheets (HCS) and corotating interaction regions (CIR). On the other hand, the $g$ value estimated from our simulation is the ratio of the scintillation index with and without CMEs at the same time 

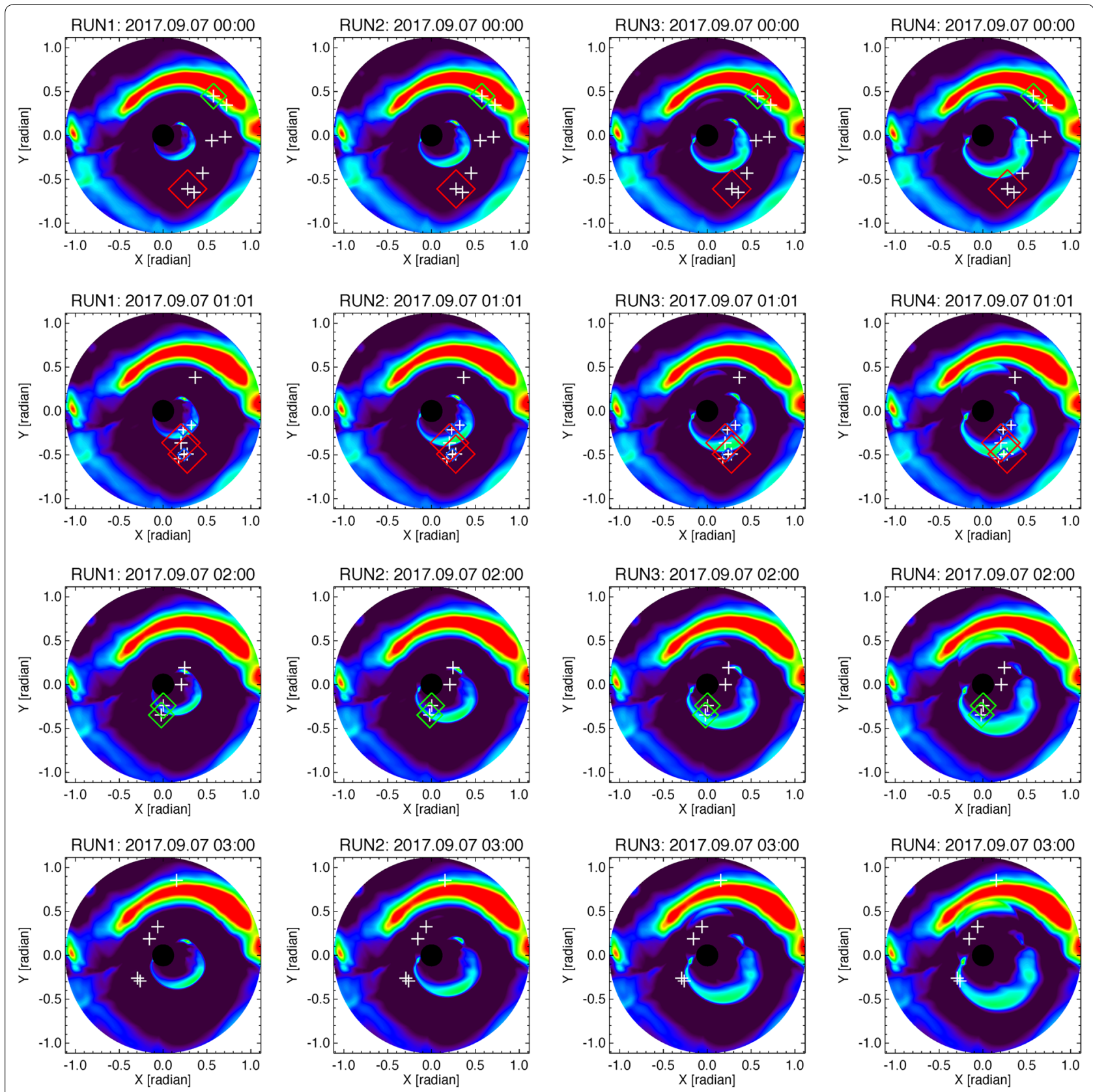

Fig. 3 Time variation of the SUSANOO-estimated (the background) and observed (symbols) IPS $g$ values on September 9, 2017. Simulations (columns from the left): RUN1, RUN2, RUN3 and RUN4. Time (rows from the top: 0:00 UT, 1:00 UT, 2:00 UT and 3:00 UT)

and direction. Therefore, our simulated $g$ values cannot be used to detect phenomena such as HCSs and CIRs that last longer than 1 day; this estimation is suitable for extracting the $g$ value enhancement generated only by CMEs. In our comparisons, the errors induced by the unavoidable inclusion of phenomena such as HCSs and CIRs in the observed $g$ values are assumed small.

\section{Spatial variation of the estimated $g$ value}

In our MHD simulation, the $g$ value enhancement shows a loop-like or toroidal distribution in agreement with the observed IPS. Figure 4 shows the density distribution on the ecliptic plane estimated by RUN2 on September 7 1:00 UT (the same time as that depicted in Fig. 2). In this analysis the enhanced $g$ value region 

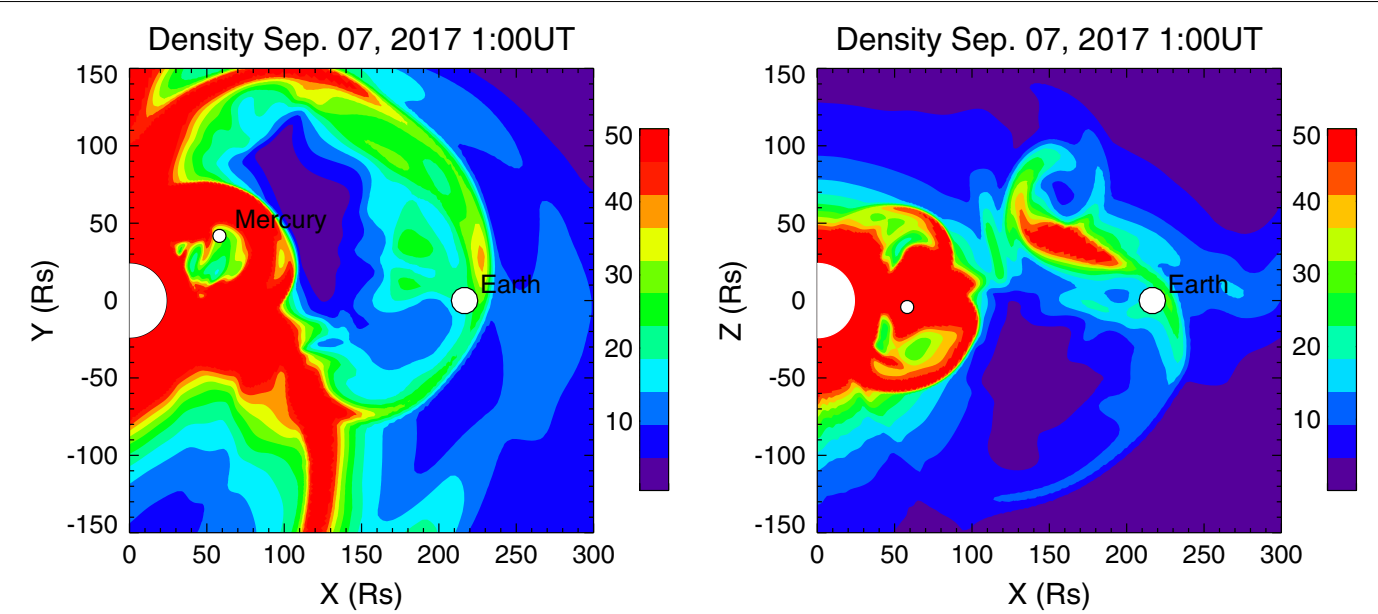

Fig. 4 (Left) Density distribution on the ecliptic plane estimated by RUN2 at 1:00 UT. (Right) Density distribution on the Sun-Earth plane inclined $90^{\circ}$ from the ecliptic plane. Location of the Earth is at $[216,0]$ as indicated by the large white circle. A smaller white circle estimates the location of Mercury projected onto these planes. Horizontal and vertical axes: distance from the Sun (Rs). Color: electron density (/cc)

is present at the front of the propagating spheromak where the density is higher than that of the surrounding solar wind. The initial spheromak does not contain any density enhancement (i.e., the density of the spheromak is equal to that of the background solar wind). The density enhancement in our simulation is generated by the compression of the solar wind swept by the higherspeed CMEs. This is consistent with the explanation given by Tokumaru et al. 2003 (see Fig. 4 in Tokumaru et al. 2003).

Figure 3 shows two groups of $g$ value enhancementsone in the northern hemisphere caused from the first $\mathrm{CME}$ and the other in the southern hemisphere caused from the second CME. Although the simulation in Fig. 4 shows that the first CME had already arrived at the Earth, a "backside lobe" of the spheromak still remains near the Earth at this time and caused the $g$ value enhancement. If we adopt the traditional assumption that the CME should be at the P-point, which is the closest point along the line of sight of the radio source, the first CME should be located between 0.5 and 0.8 $\mathrm{AU}$ at the time depicted in Fig. 4. Without this figure, we might mistake that the high $g$ values in the northern hemisphere are caused by the second CME. Our MHDbased IPS simulation shows that we can clearly distinguish the $g$ value enhancements originating from the first and second CMEs. Previously, it has been difficult to distinguish the IPS enhancements generated by multiple CMEs using all-sky maps with 1-day cadence. The IPS simulation developed in this study can be applied to studies of multiple CME events, such as CME-CME interactions, in future studies.

\section{Best CME velocity}

As shown in the previous sections, the simulated and observed $g$ values were derived using different approximations. This may present differences in the absolute $g$ values. Therefore, in further certification of our simulations, we use the location of the shock front of the CME to fit the CME speed. Figure 5 shows the relationship between the observed and estimated $g$ values along a specific declination angle from the solar north pole. At 1:00 UT, two high $g$ value sources (red lines in Fig. 5a) are observed. The shock of RUN2, RUN3 and RUN4 crossed at least 1 radio source with a high $g$ value. Weak $g$ value sources that correspond to the backside of the shock (blue lines in Fig. 5b) are also observed. At 2:00 UT, the shock of RUN3 and RUN4 has already passed the high $g$ value sources (green lines in Fig. 5c), while that of RUN1 and RUN2 is just crossing these sources. At 3:00 UT, the shock of RUN4 is just crossing a radio source without a high $g$ value (black line in Fig. 5 d). It seems that RUN2 or RUN3 fit the observed $g$ values best although both of them are not the perfect fits.

In order to provide a more objective determination of the best fit to the observed $g$ value, we use simple indices corresponding to the number of observed radio sources whose $g$ value is consistently estimated by MHD simulation. We define that the $g$ value is consistently estimated such that both estimated and observed $g$ values are larger than 1 (true positive: TP), or both of them are smaller than or equal to 1 (true negative: $\mathrm{TN})$. The accuracy of the fitting is defined as the ratio between the number of consistently estimated radio source $(\mathrm{TP}+\mathrm{TN})$ and total number of the observed radio sources around the CME. We also use a skill 
a

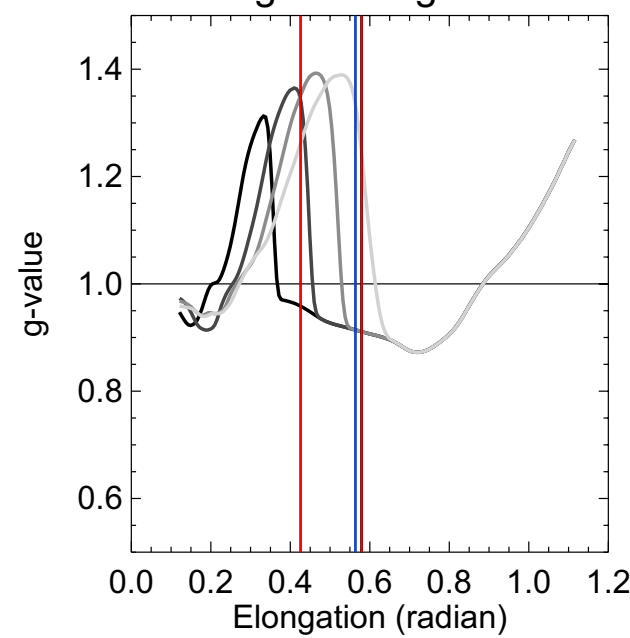

c IPS along 177-deg at 2:00 UT

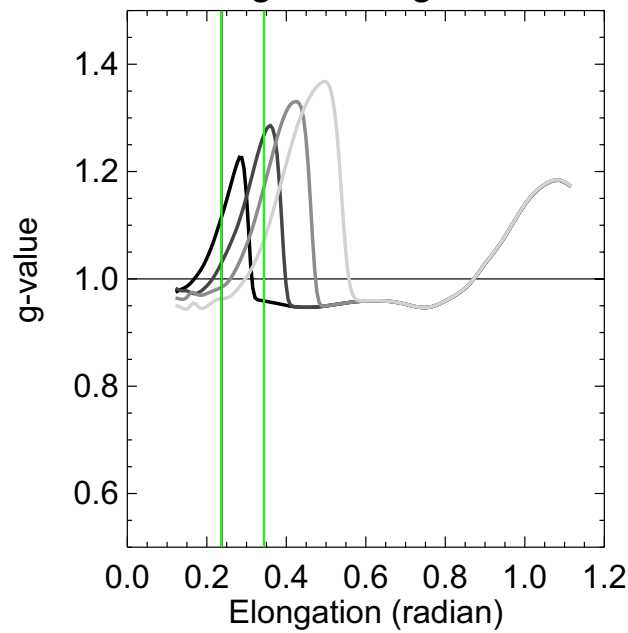

b IPS along 235-deg at 1:00 UT

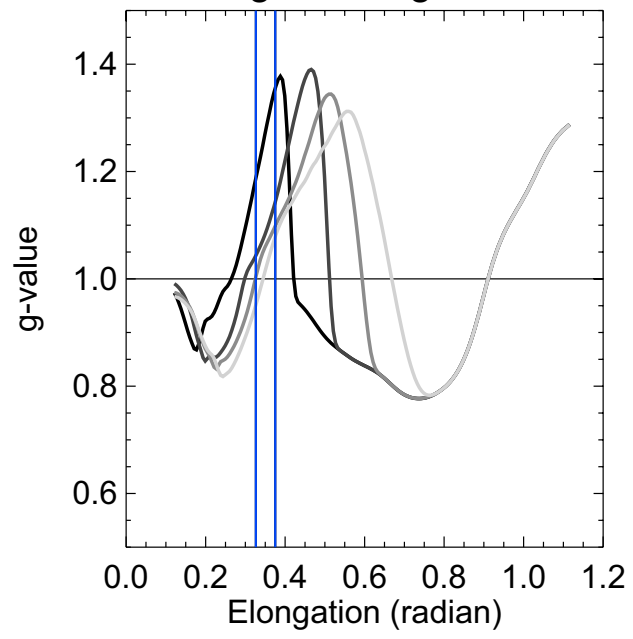

d IPS along 135-deg at 3:00 UT

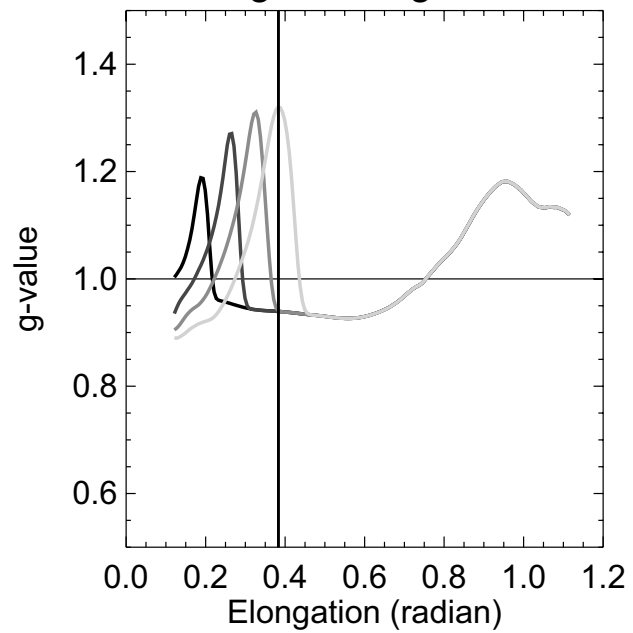

Fig. 5 Slices of the estimated $g$ value along a specific position angle from the solar north pole indicated by the solid lines in Fig. $2 \mathrm{~d}$. a Along $207^{\circ}$ at 1:00 UT, b along $235^{\circ}$ at 2:00 UT, c along $177^{\circ}$ at 2:00 UT and $\mathbf{d}$ along $135^{\circ}$ at 3:00 UT. RUN1, RUN2, RUN3 and RUN4 are indicated by the black to gray lines. Vertical lines indicate the elongation of nearby radio sources along the slice observed at the same time: red: $2.0<g$, green: $1.5<g<2.0$, blue: $1.2<g<1.5$ and black $g<1.0$

score that finds whether $g$ value is inconsistently estimated; the MHD estimates $g>1$, while the observed $g$ value was $g \leq 1$ (false positive: FP), and the MHD estimated $g \leq 1$, while the observed $g$ value was $g>1$ (false negative: FN). The true skill statistic (TSS) is defined, $\mathrm{TSS}=\mathrm{TP} /(\mathrm{TP}+\mathrm{FN})-\mathrm{FP} /(\mathrm{FP}+\mathrm{TN})$. We also evaluate the average of the positional difference between the $g$ value peak and radio sources with $g>1$ along the same position angle. Table 2 summarizes the indices of the four RUNs. RUN2 provides the best match among them.

Figure 6 shows the time-distance plot of the velocity along the Sun-Earth line in RUN1, RUN2, RUN3 and
RUN4. The two CMEs are recognized as two propagating high-speed regions. The arrival time of the second CME is defined by the shock arrival time observed by

Table 2 Initial speed (second CME) and the observed radio sources with consistent $g$ values from MHD simulation

\begin{tabular}{lllll}
\hline & RUN1 & RUN2 & RUN3 & RUN4 \\
\hline CME speed & $1000 \mathrm{~km} / \mathrm{s}$ & $1500 \mathrm{~km} / \mathrm{s}$ & $2000 \mathrm{~km} / \mathrm{s}$ & $2500 \mathrm{~km} / \mathrm{s}$ \\
Accuracy & 0.6 & 0.8 & 0.6 & 0.5 \\
TSS & 0.4 & 0.7 & 0.4 & -0.3 \\
$\begin{aligned} \text { Average differ- } \\
\text { ence (radian) }\end{aligned}$ & 0.60 & 0.55 & 0.65 & 0.75 \\
\hline
\end{tabular}


the Deep Space Climate ObseRvatory (DSCOVR) spacecraft, an in situ instrument at the Lagrange point (L1: 213 Rs), which is indicated by the horizontal line in Fig. 6 . The arrival time of the CME in RUN1, RUN2, RUN3 and RUN4 is around 24:00 UT, 22:00 UT, 18:00 TU and 14:00 UT on September 7, respectively. The actual CME shock arrived at the Earth around 22:28 UT on September 7, 2017 (Shen et al. 2018), and is indicated by the vertical line in Fig. 6 . Therefore, RUN2 is the best fit and this result is consistent with the best fit of the estimated IPS as shown in Fig. 5 and Table 2. In the CME list automatically generated by CACTus (Robbrecht et al. 2009), the median velocity and highest velocity of the second CME are 978 and $1955 \mathrm{~km} / \mathrm{s}$, respectively. These CME velocities correspond to RUN1 and RUN3, respectively. Therefore, our results suggest that the initial speed of the CME automatically derived by the white-light coronagraph images is validated by the IPS data.

\section{Concluding remarks}

We have developed an IPS estimation system based on the global MHD simulation SUSANOO-CME. The scintillation index was estimated using the density distribution in the inner heliosphere derived by the MHD simulation. Then, the $g$ value enhancement by CMEs was estimated by using the ratio of the scintillation index with and without CMEs. The simulated IPS values are compared with the IPS observations made by ISEE, Nagoya University. The simulated $g$ values show good agreement with the observed $g$ value. We simulated several $g$ value time variations generated corresponding to different initial speeds of the CME. We found that the CME that shows the best fit to the IPS
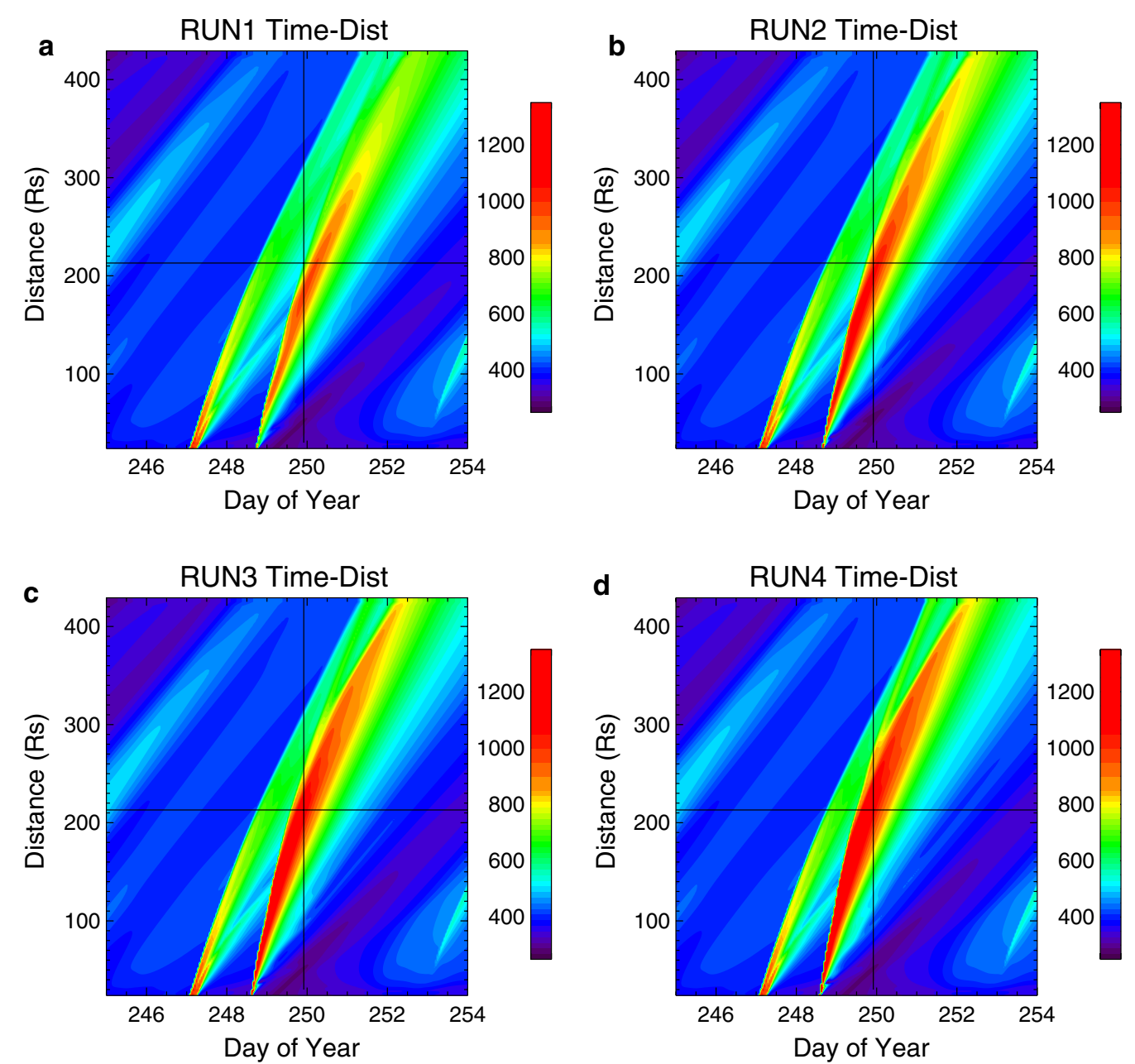

Fig. 6 Time-distance plot of the velocity derived from the SUSANOO-CME along the Sun-Earth line. Vertical axis: distance from the Sun (RS). Horizontal axis: day of year in 2017. Color contour: plasma velocity (km/s). a RUN1, b RUN2, c RUN3 and d RUN4. The horizontal line at 216 Rs indicates the location of the Earth, and the vertical line at 22:30 UT on September 7, 2017, indicates the shock arrival time 
observations allows a forecast of the arrival time of the CME at the Earth most accurately. Although a quantitative assessment of the accuracy of this system is beyond the scope of this study, our result suggests that the accuracy of the CME arrival time could be improved if the current MHD simulations include IPS data.

From the viewpoint of space weather forecasting, stable real-time predictions are important. Our MHD simulation system based on the IPS observation has a large advantage to the stable operation of the forecasting system because the ground-based IPS observations are more stable compared to the space-based observations. This system is included in the CME forecasting system developed by Shiota et al. (in prep). In their system, the initial condition of CMEs (parameters of the spheromak) is roughly derived semiautomatically from the space-based coronagraph observations and the location of the associated flare on the solar surface. Then, many CMEs with possible parameter sets are simulated using SUSANOO. Each simulation result is evaluated by the IPS observations using our IPS simulation system. This real-time forecasting system is expected to improve significantly the accuracy of the arrival time of the CME to the Earth.

\section{Abbreviations \\ CIR: corotating interaction region; CME: coronal mass ejection; HCS: helio- spheric current sheet; IPS: interplanetary scintillation; LASCO: Large Angle and Spectrometric Coronagraph; MHD: magnetohydrodynamic; SOHO: Solar and Heliospheric Observatory; SUSANOO: Space-weather-forecast-Usable System Anchored by Numerical Operations and Observations; SWIFT: Solar Wind Imaging Facility.}

\section{Authors' contributions}

$\mathrm{KI}$ led this study and drafted the manuscript. DS developed the MHD simulation codes. KI, MT and KF operated and maintained the IPS radio observations. DS, MD and YK operated and maintained the space weather forecasting system based on the MHD simulation. All authors read and approved the final manuscript.

\section{Author details \\ ${ }^{1}$ Institute for Space-Earth Environmental Research, Nagoya University, Furo-cho, Chikusa-ku, Nagoya 464-8601, Japan. ${ }^{2}$ National Institute of Infor- mation and Communications Technology, 4-2-1 Nukui-kita, Koganei, Tokyo 184-8795, Japan.}

\section{Acknowledgements}

This work was supported by MEXT/JSPS KAKENHI Grant Number 18H04442, $15 \mathrm{H} 05813$ and $15 \mathrm{H} 05814$. The IPS observations were provided by the solar wind program of the Institute for Space-Earth Environmental Research (ISEE). This study was carried by using the computational resource of the Center for Integrated Data Science, ISEE, Nagoya University, through the joint research program. We thank the LASCO coronagraph group for the white-light CME images.

\section{Competing interests}

The authors declare that they have no competing interests.

\section{Availability of data and materials}

The datasets supporting the conclusions of this article are available in the data repository of ISEE, Nagoya University http://stsw1.isee.nagoya-u.ac.jp/ ips_data-e.html, and Virtual Solar Observatory https://sdac.virtualsolar.org/cgi/ search.
Consent for publication

Not applicable.

Ethics approval and consent to participate

Not applicable.

\section{Funding}

This work was supported by the Ministry of Education, Culture, Sports, Science and Technology (MEXT), Japan Society for the Promotion of Science (JSPS), KAKENHI Grant Numbers 18H04442, $15 \mathrm{H} 05813$ and 15H05814.

\section{Publisher's Note}

Springer Nature remains neutral with regard to jurisdictional claims in published maps and institutional affiliations.

Received: 15 November 2018 Accepted: 26 March 2019

Published online: 04 April 2019

\section{References}

Arge CN, Pizzo VJ (2000) Improvement in the prediction of solar wind conditions using near-real time solar magnetic field updates. J Geophys Res 105:10465-10480. https://doi.org/10.1029/1999JA000262

Brueckner GE, Howard RA, Kooman MJ, Korendyke CM, Michels DJ, Moses JD, Socker DG, Dere KP, Lamy PL, Llebaria A et al (1995) The large angle spectroscopic coronagraph (LASCO). Sol Phys 162:357. https://doi. org/10.1007/BF00733434

Cargill PJ (2004) On the aerodynamic drag force acting on interplanetary coronal mass ejections. Sol Phys 221:135. https://doi.org/10.1023/ B:SOLA-0000033366-10725-a2

Chen J (1996) Theory of prominence eruption and propagation: interplanetary consequences. J Geophys Res 101(A12):27499. https://doi. org/10.1029/96JA02644

Gapper GR, Hewish A, Purvis A, Duffet-Smith PJ (1982) Observing interplanetary disturbances from the ground. Nature 296:633-636

Glyantsev AV, Tyul'bashev SA, Chashei IV, Shishov VI (2015) Interplanetary-scintillation observations of coronal mass ejections near the maximum of the 24th solar-activity cycle. Astron Rep 59(1):40-47. https://doi.org/10.1134/ S1063772915010047

Gopalswamy N, Lara A, Yashiro S, Kaiser ML, Howard RA (2001) Predicting the 1-AU arrival times of coronal mass ejections. J Geophys Res 106(A12):29207. https://doi.org/10.1029/2001JA000177

Hayashi K, Kojima M, Tokumaru M, Fujiki K (2003) MHD tomography using interplanetary scintillation measurement. J Geophys Res 108(A3):1102. https://doi.org/10.1029/2002JA009567

lju T, Tokumaru M, Fujiki K (2013) Radial speed evolution of interplanetary coronal mass ejections during solar cycle 23. Sol Phys 288:331. https:// doi.org/10.1007/s11207-013-0297-5

lju T, Tokumaru M, Fujiki K (2014) Kinematic properties of slow ICMEs and an interpretation of a modified drag equation for fast and moderate ICMEs. Sol Phys 289:2157. https://doi.org/10.1007/s11207-014-0472-3

Jackson BV, Hick PL, Kojima M, Yokobe A (1998) Heliospheric tomography using interplanetary scintillation observations. 1 Combined Nagoya and Cambridge observations. J Geophys Res 103:12049. https://doi. org/10.1029/97ja02528

Jackson BV, Hick PP, Buffington A, Kojima TM, Fujiki K, Ohmi T, Yamashita M (2003) Time-dependent tomography of hemispheric features using interplanetary scintillation (IPS) remote-sensing observations, in Velli, M., Bruno, R, and Malara, F. Solar Wind Ten 679:75-78

Jackson BV, Hick PP, Buffington A, Bisi MM, Clover JM, Tokumaru M, Fujiki K (2011) Three-dimensional reconstruction of heliospheric structure using iterative tomography: a review. J Atmos Sol Terr Phys 73:1214. https://doi. org/10.1016/j.jastp.2010.10.007

Jackson BV, Odstrcil D, Yu H-S, Hick PP, Buffington A, Mejia-Ambriz JC, Kim J, Hong S, Kim Y, Han J, Tokumaru M (2015) The UCSD IPS solar wind boundary and its use in the ENLIL 3D-MHD prediction model. Space Weather 13:104-115. https://doi.org/10.1002/2014SW001130 
Johri A, Manoharan PK (2016) An intense flare-CME event in 2015: propagation and interaction effects between the Sun and Earth's orbit. Sol Phys 291(5):1433-1446. https://doi.org/10.1007/s11207-016-0900-7

Kageyama A, Sato T (2004) "Yin-Yang grid": an overset grid in spherical geometry. Geochem Geophys Geosyst 5:Q09005. https://doi.org/10.1029/2004G C000734

Kojima M, Tokumaru M, Watanabe H, Yokobe A, Jackson BV, Hick PL (1998) Heliospheric tomography using interplanetary scintillation observations: 2. Latitude and heliocentric distance dependence of solar wind structure at 0.1-1 AU. J Geophys Res. https://doi.org/10.1029/97ja02162

Manoharan PK (2006) Evolution of coronal mass ejections in the inner heliosphere: a study using white-light and scintillation images. Solar Phys 235:345. https://doi.org/10.1007/s11207-006-0100-y

Möstl C, Isavnin A, Boakes PD, Kilpua EKJ, Davies JA, Harrison RA et al (2017) Modeling observations of solar coronal mass ejections with heliospheric imagers verified with the Heliophysics System Observatory. Space Weather 15:955-970. https://doi.org/10.1002/2017SW001614

Odstrcil D (2003) Modeling 3-D solar wind structure. Adv Space Res 32:497506. https://doi.org/10.1016/S0273-1177(03)00332-6

Robbrecht E, Berghmans D, Van der Linden RAM (2009) Automated LASCO CME catalog for solar cycle 23: are CMEs scale invariant? Astrophys J 691(2):1222-1234. https://doi.org/10.1088/0004-637X/691/2/1222

Rollett T, Möstl C, Isavnin A, Davies JA, Kubicka M, Amerstorfer UV, Harrison RA (2016) ElEvoHI: a novel CME prediction tool for heliospheric imaging combining an elliptical front with drag-based model fitting. Astrophys J 824:131. https://doi.org/10.3847/0004-637X/824/2/131

Shen C, Xu M, Wang Y, Chi Y, Luo B (2018) Why the shock-ICME complex structure is important: learning from the early 2017 September CMEs. Astrophys J 861:28. https://doi.org/10.3847/1538-4357/aac204

Shiota D, Kataoka R (2016) Magnetohydrodynamic simulation of interplanetary propagation of multiple coronal mass ejections with internal magnetic flux rope (SUSANOO-CME). Space Weather 14:56-75. https://doi. org/10.1002/2015SW001308

Shiota D, Kataoka R, Miyoshi Y, Hara T, Tao C, Masunaga K, Futaana Y, Terada N (2014) Inner heliosphere MHD modeling system applicable to space weather forecasting for the other planets. Space Weather 12:187-204. https://doi.org/10.1002/2013SW000989

Tokumaru M, Kojima M, Fujiki K, Yokobe A (2000) Three-dimensional propagation of interplanetary disturbances detected with radio scintillation measurements at $327 \mathrm{MHz}$. J Geophys Res 105(A5):10435. https://doi. org/10.1029/2000JA900001

Tokumaru M, Kojima M, Fujiki K, Yamashita M, Yokobe A (2003) Toroidal-shaped interplanetary disturbance associated with the halo coronal mass ejection event on 14 July 2000. J Geophys Res 108(A5):1220. https://doi. org/10.1029/2002JA009574

Tokumaru M, Kojima M, Fujiki K, Yamashita M (2006) Tracking heliospheric disturbances by interplanetary scintillation. Nonlinear Process Geophys 13:329

Tokumaru M, Kojima M, Fujiki K, Maruyama K, Maruyama Y, Ito H, lju T (2011) A newly developed UHF radiotelescope for interplanetary scintillation observations: Solar Wind Imaging Facility. Radio Sci 46:RS0F02. https:// doi.org/10.1029/2011rs004694

Vršnak B, Gopalswamy N (2002) Influence of the aerodynamic drag on the motion of interplanetary ejecta. J Geophys Res 107(A2):1019. https://doi. org/10.1029/2001JA000120

Woo R, Armstrong JW (1979) Spacecraft radio scattering observations of the power spectrum of electron density fluctuations in the solar wind. J Geophys Res 84:7288. https://doi.org/10.1029/JA084iA12p07288

Yashiro S, Gopalswamy N, Michalek G, St. Cyr OC, Plunkett SP, Rich NB, Howard RA (2004) A catalog of white light coronal mass ejections observed by the SOHO spacecraft. J Geophys Res 109:A07105. https://doi. org/10.1029/2003ja010282

Young AT (1971) Interpretation of interplanetary scintillations. Astrophys J 168:543. https://doi.org/10.1086/151108

Yu H-S, Jackson BV, Hick PP, Buffington A, Odstrcil D, Wu C-C, Davies JA, Bisi MM, Tokumaru M (2015) 3D reconstruction of interplanetary scintillation (IPS) remote-sensing data: global solar wind boundaries for driving 3D-MHD models. Sol Phys 290(9):2519-2538. https://doi.org/10.1007/ s11207-015-0685-0

\section{Submit your manuscript to a SpringerOpen ${ }^{\circ}$ journal and benefit from:}

- Convenient online submission

- Rigorous peer review

- Open access: articles freely available online

- High visibility within the field

Retaining the copyright to your article

Submit your next manuscript at springeropen.com 